\title{
THE BUSINESS JUDGMENT RULE EN LOS ESTADOS UNIDOS: UNA REGLA CON DIMENSIÓN PROCESAL Y FUERZA SUSTANTIVA*
}

\section{THE BUSINESS JUDGMENT RULE IN AMERICA: A RULE WITH PROCEDURAL DIMENSION AND SUBSTANTIVE FORCE}

\author{
Felipe Suescún De Roa** \\ Fecha de recepción: 15 de abril del 2013 \\ Fecha de aceptación: 17 de mayo del 2013
}

\section{Para citar este artículo / To cite this article}

\author{
Suescún De Roa, Felipe, The business judgment rule en los estados uni- \\ dos: una regla con dimensión procesal y fuerza sustantiva, 127 Vniversitas, \\ 341-371 (2013) \\ doi:10.1144/Javeriana.VJ127.bjre
}

* Artículo de investigación y reflexión por parte del autor en cuanto al tema central que versa sobre la responsabilidad de los administradores.

** Abogado de la Universidad de los Andes con Especialización en Derecho Comercial de la misma universidad. Maestría en Derecho (LL.M.) de Georgetown University Law Center. Socio en Suescún Abogados. Profesor de cátedra de la Universidad de los Andes. Fue asociado extranjero en el Grupo de Arbitraje Internacional de Freshfields Bruckhaus Deringer LLP. Ha publicado diversos artículos, tanto en inglés como en español, en publicaciones nacionales e internacionales en aspectos relativos a derecho comercial, corporativo, arbitraje nacional e internacional, obligaciones, contratos y derecho de los negocios en general. Contacto: felipesuescun@yahoo.com 


\section{RESUMEN}

En Delaware, en los Estados Unidos, la Business Judgment Rule (BJR) ha sido descrita como "una presunción de que en la toma de una decisión de negocios los administradores de una compañía actuaron de manera informada, de buena fe y en la creencia sincera de que la medida adoptada era en el mejor interés de la compañía". La parte que busque impugnar la decisión deberá rebatir la aplicación de la mencionada presunción. En el evento en que logre rebatirla, se invertirá la carga de la prueba, de manera que serán los administradores quienes deberán probar el "entire fairness" de la transacción.

Este artículo asimismo explica tres justificaciones de la BJR, al igual que algunas críticas a la regla basadas o relacionadas con un análisis de costo - beneficio. De otra parte, este artículo se enfoca en la aplicación de la BJR en temas bancarios, y particularmente en casos relacionados con operaciones de fusiones y adquisiciones (M\&A, por sus siglas en inglés).

Palabras clave autor: Regla del buen juicio empresarial, administradores, junta directiva, deberes fiduciarios, debida diligencia, lealtad, buena fe, deberes secundarios, responsabilidad de los administradores, acciones contra los administradores, fusiones y adquisiciones, adquisiciones hostiles, bancos, derecho bancario, administradores de bancos.

Palabras clave descriptor: Buena fe (Derecho), debido proceso de derecho, responsabilidad administrativa, derecho bancario.

1 Aronson v. Lewis, 473 A.2d 805 (Del. 1984). 


\section{ABSTRACT}

In Delaware, in the United States, the Business Judgment Rule (BJR) has been described as "a presumption that in making a business decision the directors of a company acted on an informed basis, in good faith and in the honest belief that the action taken was in the best interest of the company". The party seeking to challenge the decision must rebut the application of that presumption. In the event that the party achieves rebut, the burden of proof will be reversed, so that it will be the administrators who must prove the "entire fairness" of the transaction. This article also discusses three justifications for BJR, like some criticism of the rule based on or associated with a cost-benefit analysis. Furthermore, this article focuses on the application of BJR on banking issues, particularly in cases involving mergers and acquisitions ( $\mathrm{M} \& \mathrm{~A})$.

Keywords author: Good business judgment rule, directors, board of directors, fiduciary duties, due diligence, loyalty, good faith, secondary duties, directors' liability, actions against directors, mergers and acquisitions, hostile takeovers, banks, banking law, bank managers.

Keywords plus: Good faith (Law), due process of law, administrative responsibility, banking law.

\section{SUMARIO}

INTRODUCCIÓN.- I. THE BUSINESS JUDGMENT RULE EN LOS ESTADOS UNIDOS.- $A$. ¿Qué sucede cuando la regla no aplica?.- B. El entire fairness como estándar de revisión.- C. Efectos de una decisión interesada.- D. Consecuencias de una decisión desinformada.- E. Requisitos adicionales para la aplicación de la BJR en operaciones de $M \& A$.- F. El requisito de la buena fe.- G. Diferencia entre la BJR y el criterio de la debida diligencia.- H. Justificación de la regla.- I. Estimular a los administradores a ejercer su cargo y a tomar riesgos.- J. Evitar el escrutinio judicial.- K. Conservar a la junta directiva como último órgano de toma de decisión.- L. Críticas a la regla.- M. Aplicación de la BJR a administradores de bancos.- CONCLUSIÓN. 


\section{INTRODUCCIÓN}

Los administradores de una compañía tienen la responsabilidad de supervisar los distintos aspectos y negocios relativos a la misma ${ }^{2}$, para lo cual tienen que cumplir, principalmente, con tres deberes fiduciarios o de confianza, a saber: debida diligencia, buena fe y lealtad ${ }^{3}$. Cuando una decisión de negocios no resulta adecuada para los intereses de los accionistas, estos pueden interponer una acción de responsabilidad contra los administradores de la misma por incumplir sus deberes fiduciarios ${ }^{4}$. Generalmente, a través de este tipo de demandas los accionistas acusan a la junta directiva de haber adoptado decisiones que una persona prudente en circunstancias similares no hubiera tomado ${ }^{5}$. Sin embargo, siempre que se cumplan con ciertos requisitos, the Business Judgment Rule (en adelante BJR) protege a los administradores de no ser declarados responsables por las consecuencias que puedan ocasionar dichas decisiones.

Muchos abogados, incluso aquellos que no tienen ninguna relación con temas corporativos, probablemente han oído mencionar la BJR. No obstante lo anterior, a la gran mayoría de estos, incluso a algunos que son experimentados en dicho campo, les cuesta trabajo describirla como una regla y, en cambio, señalan que es un mecanismo para evitar que las cortes interfieran en las decisiones de negocios adoptadas por los administradores de una compañía. Si bien dicha afirmación es verdadera, no es menos cierto que se trata de un efecto de la aplicación de la BJR y no de la regla como tal.

En aras de despejar esa y otras dudas, este artículo en la primera parte describe, de manera general, la BJR en los Estados Unidos. En el segundo apartado se indican los efectos de no aplicar la BJR y se hace énfasis en el estándar de revisión que en ausencia de la BJR se aplica. Después se mencionan algunos requisitos adicionales que se han establecido para que aplique la BJR en casos relativos a fu-

2 J. R. Macey \& Maureen O'Hara, The Corporate Governance of Banks, Fed. Res. Bank of N.Y. Econ. Pol'y R. 91, 92-94 (2003).

3 Cede v. Technicolor Inc., 634 A. 2d 361 (Del. 1993).

4 J. R. Macey \& Maureen O'Hara, The Corporate Governance of Banks, Fed. Res. Bank of N.Y. Econ. Pol'y R. 91, 93 (2003).

5 El estándar de la debida diligencia adoptado por las cortes en Delaware es el de "una persona prudente en circunstancias similares". M. McMurray, Historical Perspective on the Duty of Care, the Duty of Loyalty, and the Business Judgment Rule, 40 Vand. L. Rev., 607 (1987). 
siones y adquisiciones (en adelante, por sus siglas en inglés, M\&A). Posteriormente se hace alusión brevemente al requisito de la buena fe y a las diferencias entre la BJR y el deber de diligencia. Adicionalmente, se señalan distintas teorías que justifican la adopción de dicha regla, al igual que algunas de las críticas respecto de la misma. El artículo continúa haciendo una breve descripción de distintos casos relacionados con la aplicación de la regla a administradores de bancos. Finalmente, termina concluyendo que no obstante la BJR no ha sido adoptada en muchos países del derecho europeo continental, un gran número de criterios utilizados por las cortes de los Estados Unidos en relación con ella resultan ser aplicables en dichos países y, sin duda, sirven para orientar a jueces, árbitros y abogados en casos relacionados con acciones de responsabilidad contra los administradores

\section{THE BUSINESS JUDGMENT RULE EN LOS ESTADOS UNIDOS}

La Corte Suprema de los Estados Unidos, en el caso Edgar v. MITE Corp., estableció que los aspectos de derecho corporativo, "específicamente aquellos que envuelven relaciones fiduciarias entre administradores y accionistas" " 6 , han de ser gobernados por la ley del lugar de constitución de la compañía. En el estado de Delaware, en los Estados Unidos, están constituidas más de 945.000 compañías, incluyendo el 63\% de las 500 empresas más grandes de dicho país por ingresos brutos?

Además de ser Delaware el "hogar legal" de un gran número de compañías norteamericanas, dicho estado es reconocido por tener la legislación más avanzada en materia de derecho corporativo de los Estados Unidos, al igual que por tener los "jueces mejor preparados" en dicha materia y porque sus jueces tienen "un historial de producir resultados racionales" $"$. De este modo, otras cortes de los Estados Unidos, al resolver aspectos complicados relativos a derecho de los negocios, frecuentemente hacen referencia a decisiones de

\footnotetext{
E. MITE Corp., 457 U.S. 624, 645 (1982).

Delaware Division of Corporations. Disponible en: http://www.state.de.us/corp

L.E. Strine, Jr., Delaware's Corporate-Law System: Is Corporate America Buying an Exquisite Jewel or a Diamond in the Rough? A Response to Kahan \& Kamar's Price Discrimination in the Market for Corporate Law, 86 Cornell L. Rev., 1257, 1263 (2001).
} 
cortes en Delaware, pues dichas cortes tienen la reputación de ser las "cortes madre del derecho corporativo" 9 en los Estados Unidos.

La BJR ha existido por cerca de dos siglos ${ }^{10}$ y ha sido prácticamente adoptada a través de todo Estados Unidos ${ }^{11}$. Otros países del common law, sin embargo, tales como el Reino Unido, no lo han hecho. La visión general de los ingleses a este respecto es que no existe la necesidad de adoptar dicha regla, toda vez que existen principios tanto del common law como contenidos en su legislación que protegen las decisiones comerciales y de negocios adoptadas por los administradores de las compañías de la excesiva intromisión de las cortes. De este modo, para evaluar la posibilidad de que las cortes inglesas revisen decisiones comerciales y de negocios de los administradores es necesario analizar el alcance de los deberes de los mismos de acuerdo con la ley inglesa (Companies Act 2006) ${ }^{12}$.

En Delaware la BJR ha sido descrita como "una presunción de que en la toma de una decisión de negocios los administradores de una compañía actuaron de manera informada, de buena fe y en la creencia sincera de que la medida adoptada era en el mejor interés de la compañia"13.

Existe una segunda formulación de la BJR, la cual ha sido adoptada por el American Law Institute (ALI) en sus Principles of Corporate Governance. Dichos principios comienzan señalando que los administradores de una compañía tienen frente a la misma la obligación de actuar de buena fe, en una forma que estos razonablemente crean que es en el mejor interés de la compañía, y con la diligencia que razonablemente se esperaría que ejerciera una persona prudente en una posición similar y bajo circunstancias similares ${ }^{14}$. Los principios también señalan que un administrador que toma una decisión de buena fe cumple con el deber de diligencia si: (1) no tiene un interés personal en la decisión de negocios; (2) está debidamente informado; y (3) razonablemente cree que la decisión

9 K. v. Kemper Fin. Servs., 908 F.2d 1338, 1343 (7th Cir. 1990).

10 P. V. Letsou, Implications of Shareholder Diversification on Corporate Law and Organization: The Case of the Business Judgment Rule, 77 Chi.-Kent L. Rev., 179 (2001).

11 D. C. Langevoort, The Human Nature of Corporate Boards: Law, Norms, and the Unintended Consequences of Independence and Accountability, 89 Geo. L.J., No. 797, 818 (2001).

12 S. Worthing, Reforming Director's Duties, 64 Modern Law Review, No. 3, 439 (2001).

13 A. v. Lewis, 473 A.2d 805, 812 (Del. 1984).

14 American Law Institute, Principles of Corporate Governance: Analysts and Recommendations, at $\S 4.01$ (a) (1994) (Principles). 
de negocios ha sido tomada en el mejor interés de la compañía ${ }^{15}$. Consecuentemente, si las condiciones para la aplicación de la regla son cumplidas, no hay posibilidad de que proceda una demanda contra los administradores sustentada en una violación al deber de diligencia. Adicionalmente, los principios disponen que quien pretenda impugnar la decisión tendrá la carga de demostrar que el administrador no satisfizo los mencionados requisitos ${ }^{16}$.

No obstante las distintas interpretaciones y alcances que se le han dado tanto a la formulación de la BJR de Delaware como la de ALI, existen ciertos aspectos relacionados con la BJR que son generalmente aceptados, tales como: que esta aplica para administradores ${ }^{17}$; que la BJR protege a los administradores cuando actúan de manera colectiva, esto es, como junta directiva ${ }^{18}$; que es aplicada solo a decisiones de los administradores, no a omisiones de los mismos 19 ; que protege a administradores "desinteresados", esto es, aquellos que no sean parte de la transacción ni que puedan esperar que se derive para ellos un beneficio económico ${ }^{20}$; que la regla únicamente es aplicada para decisiones que hayan sido tomadas con la debida información, de manera que los administradores tienen la obligación de considerar "toda la información material razonablemente a su alcance" 21 ; que la regla no está destinada a proteger a administradores que han cesado en sus funciones o que no hayan actuado de buena $\mathrm{fe}^{22}$; que la BJR protege decisiones que hayan sido adoptadas de acuerdo con el deber de lealtad ${ }^{23}$; que la regla aplica cuando los administradores actúan con la debida diligencia $^{24}$, la cual es medida con el estándar de la culpa grave, no la simple negligencia ${ }^{25}$; que la regla es aplicable en aquellos casos en que la decisión de los administradores no contraviene lo dispuesto

15 Id. at $\$ 4.01$ (c).

16 Id. at $\$ 4.01$ (d).

17 Brehm, 746 A.2d at 264 n.66; Aronson, 473 A.2d at 812.

18 Aronson, 473 A.2d at 812-13; R. Franklin Balotti \& Joseph Hinsey IV, Director Care, Conduct, and Liability: The Model Business Corporation Act Solution, 56 Bus. Law. 35, 56 (2000) (la BJR aplica a ambos, a la decisión y a quien toma la decision).

19 Aronson, 473 A.2d at 813.

20 Aronson v. Lewis, 473 A.2d 805. 812 (Del. 1984).

21 Id.

22 L. Johnson, The Modest Business Judgment Rule, 55 Bus. Law. 625. 631 n.29 (2000).

23 Hun v. Cary, 82 N.Y. 65, 70 (1880).

24 Brehm, 746 A.2d at 264 n. 66.

25 Cede \& Co. v. Technicolor, Inc., 634 A.2d 345, 364 n. 31 (Del. 1993); Smith v. Van Gorkom, 488 A.2d 858, 873 (Del. 1985). 
en los estatutos sociales de la compañía ${ }^{26}$; y, finalmente, algunos casos han señalado que la BJR no protege a administradores que intencionalmente han incurrido en una conducta ilegal ${ }^{27}$.

Así las cosas, la BJR se enfoca en los mecanismos y procedimientos utilizados por los administradores en llegar a su decisión y no en un examen ex post facto de la conveniencia y/o astucia de la misma ${ }^{28}$. De este modo, los administradores no serán responsables, y las decisiones adoptadas por estos no serán anuladas, por un simple error de juicio ${ }^{29}$.

En suma, la BJR es una formulación de distintos aspectos de hecho y de derecho que deben ser tenidos en cuenta por el juez para determinar si puede existir intervención judicial en una determinada decisión de negocios ${ }^{30}$. Únicamente cuando el juez haya concluido que todos esos prerrequisitos de hecho o de derecho han sido cumplidos cabalmente, este no entrará a revisar la decisión adoptada por los administradores. En cambio, si existe prueba de que no se ha cumplido con uno o más de los elementos requeridos para la aplicación de la BJR, el juez entrará a revisar la decisión de negocios y determinará si hay lugar a declarar la responsabilidad personal de los administradores ${ }^{31}$.

\section{A. ¿Qué sucede cuando la regla no aplica?}

Dado que la BJR protege las decisiones de los administradores del escrutinio judicial, la parte interesada en impugnar dichas decisiones tiene la carga de demostrar los hechos que puedan rebatir la aplicación de la presunción ${ }^{32}$. A menos de que el demandante pueda rebatir la presunción contenida en el BJR, la Corte no podrá

26 Patricia A. McCoy, The Notional Business Judgment Rule in Banking, 44 Catholic U. L. Rev., 1031, 1036 (1995).

27 Miller v. American Telephone \& Telegraph, 507 F.2d 759 (3rd Cir. 1974).

28 Smith v. Van Gorkom: The Business of Judging Business Judgment, 41 Bus. Law, 1187, 1190 (1986).

29 Radolv. Thomas, 772 F.2d 244, 256-57 (6th Cir. 1985).

30 David A. Drexler, Lewis S. Black, \& A. Gilchrist Sparks, Delaware Corporation Law and Practice, $\S 15.03$ (2010).

31 Id.

32 McMullin v. Beran, 765 A.2d 910, 917 (Del. 2000); Cede \& Co. v. Technicolor, Inc., 634 A.2d 345, 361 (Del. 1983). 
sustituir la decisión de los administradores siempre que dicha decisión pueda ser "atribuida a algún propósito racional de negocios"33.

Bajo la formulación de la BJR en Delaware, para dejar sin efectos la presunción contenida en dicha regla, el demandante tendrá que demostrar: que los administradores no tomaron una decisión ${ }^{34}$; que estos no eran independientes o desinteresados ${ }^{35}$; que la decisión adoptada no fue razonablemente informada ${ }^{36}$; o que las decisiones implicaban culpa grave $^{37}$.

\section{B. El entire fairness como estándar de revisión}

Si la parte que impugna la decisión logra demostrar alguno de los anteriores extremos, la carga de la prueba se invertirá de manera tal que los administradores habrán de demostrar el entire fairness (razonabilidad) de la transacción ${ }^{38}$.

Si bien el entire fairness standard fue originalmente utilizado en aquellos casos donde había sido incumplido el deber de lealtad, no es menos cierto que las cortes en Delaware lo han usado adicionalmente, como se verá más adelante, en casos de negligencia ${ }^{39}$.

Dentro del examen que llevan a cabo los jueces para determinar si una determinada transacción cumple o no con el entire fairness standard, por una parte, analizan si se trata de un fair dealing, para lo cual examinan el proceso mediante el cual se adoptó la decisión, la negociación, la estructuración de la transacción y la información proporcionada. De otra parte, el juez observa si existió un fair price, para lo cual considera no solo el precio obtenido y pagado por la transacción, sino también las implicaciones económicas y de carácter financiero envueltas en la operación ${ }^{40}$.

33 Brehm v. Eisner, 746 A.2d at 264 n. 66 (Del. 2000).

34 Kaplan v. Centex Corp., 284 A.2d 119, 124 (Del. Ch. 1971).

35 Aronson, 473 A.2d at 812.

36 Smith v. Van Gorkom, 488 A.2d 858, 870-71 (Del. 1985); In re Walt Disney Co. Derivative Litig., 825 A.2d 275, 279 (Del. Ch. 2003).

37 P. Mart Realty Co. v. Becker, 298 A.2d 349, 351 (Del.Ch.1972); Katz v. Chevron Corp., 27 Cal. Rptr.2d 681, 689 (Cal. Ct. App. 1994); Aronson, 473 A.2d at 812; Van de Walle v. Unimation, Inc., [1990-1991 Transfer Binder] Fed. Sec. L. Rep. (ссн) 95,834, at 99,030-31 (Del. Ch. Mar. 6, 1991).

38 McMullin, 765 A.2d at 917;Cede \& Co., 634 A.2d at 361;Aronson, 473 A.2d at 812.

39 Ver por ejemplo: Cede \& Co. v. Technicolor, Inc., 634 A.2d 345 (Del. 1993). En este caso, debido a que la junta directiva no actuó con la debida diligencia, la Corte Suprema de Delaware no aplicó la BJR y por tanto, juzgó la transacción de acuerdo con el entire fairness standard.

40 Weinberger v. UOP, Inc., 457 A.2d 711 (Del. 1983); Cristina Guerrero Trevijano, La Business 
En ese orden de ideas, en las acciones de responsabilidad contra los administradores, los demandantes tendrán que demostrar que aquellos no fueron diligentes o que incumplieron con el deber de lealtad, probando que la decisión adoptada por los administradores fue desinformada o que implicaba culpa grave o que estos no eran independientes. De ser demostrada la falta de diligencia o de lealtad de los administradores, se desvirtúa la presunción contenida en la BJR y estos quedarán sometidos al entire fairness standard, invirtiéndose, de este modo, la carga de la prueba. Así las cosas, para que los administradores no sean declarados responsables por la decisión que fue adoptada negligentemente o en forma desleal, tendrán que entrar a demostrar que, en cualquier caso, la decisión fue justa y que no fue en perjuicio de los accionistas.

Además de la razonabilidad de la transacción, si los administradores logran demostrar la ausencia de vínculo de causalidad entre su actuación y la pérdida o la ausencia de daños, los jueces no podrán declarar la responsabilidad de los $\operatorname{mismos}^{41}$. La decisión de los administradores en dichas circunstancias habrá de ser respetada, de manera que no serán declarados responsables personalmente ${ }^{42}$.

\section{Efectos de una decisión interesada}

De otra parte, y en relación con la independencia de los administradores, se ha señalado que un administrador es considerado como interesado cuando: (i) es parte en una transacción; (ii) tiene una relación familiar, financiera o de negocios con una de las partes en la misma; (iii) tiene un interés monetario en el negocio; o (iv) está sujeto a una influencia controlante por alguna de las partes en la transacción ${ }^{43}$.

En este punto, es del caso precisar que con que uno o dos de los miembros de la junta directiva tengan conflictos de interés no es suficiente para acabar con la presunción de independencia de

Judgment Rule en los Procesos de M\&A. Documentos de Trabajo del Departamento de Derecho Mercantil, Universidad Complutense. 18-19 (2010).

41 E. S. Miller \& T. E. Rutledge, The Duty of Finest Loyalty and Reasonable Decisions: The Business Judgment Rule in Unincorporated Business Organizations? 30 Del. J. Corp. L., 343, $352(2005)$

42 D.J. Block et ál., The Business Judgment Rule-Fiduciary Duties of Corporate Directors, 5th ed., 6 (1998).

43 Principles of Corporate Governance: Analysis and Recomendations, 1.23(A) (1994). 
toda la junta directiva ${ }^{44}$. Sin embargo, si la mayoría de los otros administradores están dominados por el o los que tienen un interés en la transacción, la totalidad de la junta directiva podría carecer de independencia ${ }^{45}$.

En el supuesto de que el demandante pueda probar que la mayoría o una parte importante de los miembros de la junta directiva estaban interesados en la transacción, necesariamente habría una violación al deber de lealtad ${ }^{46}$. De este modo, como ya se mencionó, la carga de la prueba sobre el "fairness" de la transacción recae sobre los administradores ${ }^{47}$.

Estos casos de falta de independencia se presentan, por ejemplo, dentro del contexto de las operaciones de M\&A, principalmente en situaciones de tomas de control, cuando los administradores se benefician del negocio sin que así lo hagan la sociedad ni los accionistas $^{48}$. También puede haber ausencia de independencia en este tipo de situaciones cuando la operación misma implica que entren en conflicto los intereses de los administradores con los de la sociedad o con los de los accionistas; tal sería el caso de presentarse distintas ofertas y que los administradores favorezcan una en concreto, movidos, principalmente, porque el éxito de dicha oferta les garantiza la permanencia en su cargo ${ }^{49}$. De igual forma, podría existir conflicto de interés en las llamadas squeeze-out mergers, en las que la sociedad controlante se encuentra a ambos lados de la operación, de manera que los administradores pueden tener intereses encontrados ${ }^{50}$.

44 Cede \& Co. v. Technicolor, Inc., 634 A.2d 345 (Del. 1993).

45 Paula J. Dalley, The Business Judgment Rule: What You Thought You Knew, 60 Consumer Fin L.Q. Rep. 28 (2006).

46 Id. at 25.

47 Sin embargo, en algunos casos, la carga de la prueba se invierte, de manera que será el demandante quien debe probar que la transacción fue injusta. En este sentido, en Kahn v. Lynch Commun. Sys., Inc. se señaló que la ratificación de una decisión por parte de accionistas desinteresados, en una compañía en la que existía un socio controlante, implica la inversión de la carga de la prueba, de manera que son los demandantes quienes tienen que demostrar que la transacción fue injusta. Kahn v. Lynch Commun. Sys., Inc., 638 A.2d 1210 (Del. 1994).

48 Ivanhoe Partners v. Newmont Mining Corp., 535 A.2d 1334 (Del. 1987).

49 C. Guerrero Trevijano, La Business Judgment Rule en los Procesos de M\&A. Documentos de Trabajo del Departamento de Derecho Mercantil, Universidad Complutense, 17 (2010).

50 Id. at 18 . 


\section{Consecuencias de una decisión desinformada}

Respecto de las decisiones de la junta directiva adoptadas sin suficiente información, es preciso hacer referencia a Smith v. Van Gorkom, en el que los administradores fueron declarados responsables por no estar debidamente informados en relación con una fusión entre dos compañías ${ }^{51}$. En este caso se trataba de una cashout merger, la cual es un tipo de fusión en la que se excluye a los socios minoritarios, ya que estos, en cambio de recibir acciones de la nueva sociedad fusionada (como sí lo hacen los accionistas mayoritarios) reciben dinero en efectivo ${ }^{52}$.

La Corte Suprema de Delaware rechazó la aplicación del BJR en este caso y señaló que la junta directiva había violado deberes fiduciarios, principalmente el deber de diligencia, al no haber estado debidamente informada y al no haber revelado adecuadamente la información a los accionistas en busca de un voto favorable para la fusión ${ }^{53}$. En este punto se ha de señalar que la información que deben obtener y proporcionar los administradores es aquella que razonablemente está disponible o se puede obtener y no aquella que está fuera del alcance de los mismos ${ }^{54}$.

Como consecuencia de no haber cumplido apropiadamente con el deber de información, la Corte en Smith v. Van Gorkom aplicó el entire fairness standard y determinó que en el comportamiento de los administradores de la compañía había mediado culpa grave, de manera que los declaró responsables y los condenó a pagar la diferencia entre el valor justo de mercado y el precio de las acciones que efectivamente se pagó en la fusión ${ }^{55}$.

51 Smith v. Van Gorkom, 488 A.2d 858 (Del. 1985).

52 G. Subramanian, Fixing Freezouts, 115 Yale Law Journal, 2 (2005).

53 Smith v. Van Gorkom, 488 A.2d 858 (Del. 1985).

54 Brehm v. Eisner, 746 A. 2d 244 (Del. 2000).

55 Id. Como consecuencia de Smith v. Van Gorkom, "la reacción legislativa no se hizo esperar y el legislador de Delaware promulgó la sección 102(b)(7) de la Delaware General Corporation Law (DGCL) en virtud de la cual se permitía a los accionistas incluir en los estatutos de la sociedad una cláusula garantizando inmunidad a los administradores frente a la exigencia de responsabilidad por vulneraciones del deber de diligencia. A través de esta sección de la DGCL, los estatutos podían eliminar o limitar la responsabilidad de los administradores cuando en su actuación no concurrieran una serie de circunstancias, tales como: la mala fe, ilegalidad o actuación interesada del administrador". Cristina Guerrero Trevijano, La Business Judgment Rule en los Procesos de $M \& A$. Documentos de Trabajo del Departamento de Derecho Mercantil, Universidad Complutense, 15 (2010). Así las cosas, en caso de que en los estatutos de una sociedad se incluya una cláusula garantizando inmunidad a los administradores frente a demandas como las de 
Igualmente, en Cede v. Technicolor Inc., la Corte Suprema de Delaware señaló en relación con una acción de responsabilidad en contra de los administradores por una operación de adquisición, que los demandantes habían logrado demostrar que los administradores habían incumplido con su deber de diligencia al no haberse informado adecuadamente, por lo que la BJR no era aplicable y, por tanto, la decisión debía ser revisada de acuerdo con el entire fairness standard ${ }^{56}$.

\section{E. Requisitos adicionales para la aplicación de la BJR en operaciones de M\&A}

Debido a la proliferación durante los años ochenta de las adquisiciones hostiles y a la aparición de medidas defensivas frente a las mismas $^{57}$, las cortes de Delaware, además de los requisitos que se han mencionado anteriormente, han venido añadiendo otros adicionales para que pueda ser aplicada la BJR en procesos relacionados con operaciones de M\&A.

Dichos casos han estado relacionados, principalmente, con tomas de control hostil, en particular con adopción de medidas defensivas por parte de la sociedad target al igual que con adquisiciones o cambios en las posiciones de control en las sociedades ${ }^{58}$.

Van Gorkom, los accionistas que pretendan demandar a la junta directiva y que busquen que dicha cláusula no tenga efectos, tendrán que alegar que hubo un incumplimiento al deber de lealtad o que existió mala fe por parte de los administradores. Emerald Partners v. Berlin, 726 A.2d 1215. 1223-1234 (Del. 1999). En In re Walt Disney Co. Derivative Litigation, la ausencia de buena fe de los directores no fue utilizada para rebatir la presunción contenida en la BJR, sino para rebatir la aplicación de una cláusula de inmunidad para los administradores en los términos de la sección 102(b) (7) de la DGCL. In re Walt Disney Co. Derivative Litigation, 825 A.2d 275 (Del. Ch. 2003).

56 Cede v. Technicolor Inc, 634 A.2d 371 (Del. 1993); Cristina Guerrero Trevijano, La Business Judgment Rule en los Procesos de M\&A. Documentos de Trabajo del Departamento de Derecho Mercantil, Universidad Complutense. 20 (2010). No obstante lo anterior, en Weinberger v. UOP, Inc. se aseguró que en casos donde existan tomas de control en las que se aleguen conflictos de interés, si la decisión de los administradores fue aprobada por asesores independientes o por la mayoría de accionistas minoritarios que estaban debidamente informados, el juez presumirá el entire fairness de la decisión, de manera que se invertirá la carga de la prueba, siendo los demandantes los llamados a demostrar ausencia de fairness en la transacción. Weinberger v. UOP, Inc., 457 A.2d 701 (Del. 1983).

57 C. Guerrero Trevijano, La Business Judgment Rule en los Procesos de M\&A. Documentos de Trabajo del Departamento de Derecho Mercantil, Universidad Complutense, 8 (2010).

58 Id. at 22. 
En Unocal Corp. v. Mesa Petroleum ${ }^{59}$, este último, quien poseía un 13\% de las acciones de Unocal, lanzó una Oferta Pública de Adquisición (OPA) con el fin de obtener un 37\% más de las acciones $\mathrm{y}$, posteriormente, absorber a la sociedad matriz. Como resultado de estas operaciones, los accionistas minoritarios de Unocal recibirían bonos basura de la sociedad absorbente. En respuesta a dicha situación, los administradores decidieron realizar una oferta de adquisición de acciones propias con el fin de excluir la oferta de Mesa Petroleum. De este modo, la Corte Suprema de Delaware debía resolver si la medida de defensa utilizada por los administradores podía ser considerada como una actuación diligente y si su intención había sido simplemente proteger sus propios intereses sin importar los de los accionistas. A este respecto dicha Corte señaló que la oferta de Mesa Petroleum era coercitiva e inadecuada y que los administradores no incumplieron sus deberes fiduciarios, toda vez que estos adoptaron medidas defensivas razonables contra una oferta que suponía una amenaza para los intereses de la sociedad y que su reacción fue proporcionada con la amenaza ${ }^{60}$.

En ese orden de ideas, Unocal Corp. v. Mesa Petroleum establece otros criterios adicionales para que pueda ser aplicada la BJR en casos en que sean cuestionadas decisiones de los administradores por la adopción de medidas de defensa frente a amenazas hostiles para la toma de control de la sociedad ${ }^{61}$. En primer lugar, será necesario que las medidas de defensa aprueben un test de razonabilidad, para lo cual será necesario que las decisiones sean consideradas como razonables respecto de los eventuales daños causados a la sociedad. Además, dichas decisiones tendrán que enfrentar un test de proporcionalidad en el que se tendrá que concluir que la medida defensiva era adecuada respecto de la amenaza existente ${ }^{62}$. Desde 1985, la Corte Suprema de Delaware, al igual que otras cortes de dicho estado, ha aplicado, en repetidas ocasiones, los principios

59 Unocal Corp. v. Mesa Petroleum Co., 493 A.2D 946 (Del.1985).

60 C. Guerrero Trevijano, La Business Judgment Rule en los Procesos de $M \& A$. Documentos de Trabajo del Departamento de Derecho Mercantil, Universidad Complutense, 23 (2010).

61 David A. Drexler, Lewis S. Black, \& A. Gilchrist Sparks, Delaware Corporation Law and Practice, $\$ 15.14$ (2010).

62 Hunt, P. en Structuring Mergers and Acquisitions: A Guide to Creating Shareholders Value, Wolters Kluwer, $4^{\text {th }}$ ed., 507 (2009). En: Cristina Guerrero Trevijano, La Business Judgment Rule en los procesos de $M \& A$. Documentos de Trabajo del Departamento de Derecho Mercantil, Universidad Complutense, 25 (2010). 
señalados en Unocal Corp. v. Mesa Petroleum en casos que involucran medidas de defensa ${ }^{63}$.

Otro caso de gran influencia dentro de este contexto ha sido Revlon, Inc. v. Mac Andrews \& Forbes Holdings, Inc., en el que se reconocieron las exigencias del caso Unocal, esto es, la razonabilidad y proporcionalidad. Adicionalmente, se señaló que el deber de los administradores que se enfrenten a situaciones donde existan "batallas por el control" de la compañía y que, como consecuencia, hayan adoptado medidas defensivas, es, en principio, el de preservar la compañía. Sí la venta o la desintegración de la compañía es inevitable, el deber de la junta directiva pasa de la preservación de la compañía a ser la maximización del valor de la sociedad en la venta con el fin de lograr el mayor beneficio para los accionistas ${ }^{64}$. En este mismo sentido, en Paramount Communications Inc. v. QVC Network Inc. se afirmó que en aquellas operaciones que supongan un cambio de control de la sociedad o un quebrantamiento de la misma, los administradores deberán buscar el mejor valor posible para los accionistas ${ }^{65}$.

\section{F. El requisito de la buena fe}

Como ha sido mencionado anteriormente, para que opere la BJR es necesario que los administradores hayan actuado de buena fe. En Parnes v. Bally Entertainment Corp., la Corte Suprema de Delaware afirmó que la presunción contenida en la BJR había sido rebatida por el demandante, en el entendido de que este había logrado demostrar que la junta directiva había permitido que administradores de la compañía negociaran una fusión con otra empresa a un precio injusto para los accionistas $\mathrm{y}$, adicionalmente, que dichos administradores obtuvieran pagos en su propio beneficio ${ }^{66}$. La Corte señaló que el pago a los administradores fue de tal magnitud que la

63 Ver, por ejemplo: Henley Group, Inc. v. Santa Fe Southern Pacific Corp., CA 9569, 1988 Del. Ch. Lexis 32 (Mar. 11, 1988); Paramount Commc'ns Inc. v. Time Inc., 1989 Del. Ch. Lexis 77, at *70-71 (July 14, 1989); In re Time, Inc. S'holders Litig., 571 A.2d 1140 (Del. 1989); Compare Doskocil Cos. v. Griggy, CA 10095, 10106-08, 10116, 1988 Del. Ch. Lexis 113 (Aug. 18, 1988).

64 Revlon, Inc. v. MacAndrews \& Forbes Holdings, Inc., 506 A.2d 182 (Del. 1986).

65 Paramount Communications Inc. v. QvC Network Inc., 637 A. 2 d 34 (Del. 1994); Cristina Guerrero Trevijano, La Business Judgment Rule en los procesos de M\&A. Documentos de Trabajo del Departamento de Derecho Mercantil, Universidad Complutense, 30 (2010).

66 Parnes v. Bally Entertainment Corp., 722 A.2d 1243 (Del. 1999). 
aprobación de la junta directiva no podía ser cubierta por la $\mathrm{BJR}^{67}$. Asimismo puntualizó que el proceso de fusión estaba tan contaminado que era "inexplicable que administradores independientes, actuando de buena fe, pudieran aprobar el acuerdo"68.

En Crescent/Mach I Partners, L.P. v. Turner, de igual forma, se aplicó el mismo fundamento, toda vez que la decisión adoptada por la junta directiva no fue protegida por la BJR puesto que esta aceptó que el director general de la compañía hiciera negocios en su propio beneficio ${ }^{69}$. El director general, quien estaba negociando la venta de la misma, le manifestó al comprador que él no daría su consentimiento para transacción alguna, a menos que él recibiera un tratamiento especial en la transacción ${ }^{70}$. La decisión de la junta directiva aprobando la transacción, en la que se beneficiaba directamente al director general, no fue protegida por el BJR puesto que la junta directiva no actuó de buena fe protegiendo los intereses de los accionistas ${ }^{71}$.

En ambos casos, la mayoría de la junta directiva fue considerada como independiente. Sin embargo, la Corte concluyó que se había desvirtuado la presunción contenida en la BJR al haber existido un quebrantamiento al deber de lealtad por no haber actuado los administradores de buena fe en el mejor interés de los accionistas ${ }^{72}$.

\section{G. Diferencia entre la BJR y el criterio de la debida diligencia}

En términos generales, la diferencia radica en que el estándar de la debida diligencia es el nivel de conducta esperado de los administradores en su toma de decisiones, mientras que la BJR es un estándar de revisión que permite determinar si estos pueden ser declarados responsables por una decisión imprudente ${ }^{73}$.

67 Id. at 1246.

68 Id. at 1247.

69 Crescent/Mach I Partners, L.P. v. Turner, 846 A.2d 963 (Del. Ch. 2000).

70 Id.

71 Id.

72 P.J. Dalley, The Business Judgment Rule: What You Thought You Knew, 60 Consumer Fin L.Q. Rep. 24. 27 (2006).

73 E.S. Miller \& Thomas E. Rutledge, The Duty of Finest Loyalty and Reasonable Decisions: The Business Judgment Rule in Unincorporated Business Organizations?, 30 Del. J. Corp. L., 343. 352 (2005). 
De este modo, el deber de la debida diligencia es un estándar de conducta, mientras que la BJR es un estándar de revisión ${ }^{74}$. En derecho corporativo, un estándar de revisión judicial es una expresión verbal que describe la tarea que lleva a cabo un juez para determinar si con la actuación de los administradores se violó o no alguno de sus deberes fiduciarios ${ }^{75}$. Existe una relación cercana, pero no perfecta, entre el estándar con el que las cortes revisan la responsabilidad de los administradores y el estándar de comportamiento que normativamente se espera de los mismos ${ }^{76}$. Por una parte, un estándar de conducta establece cómo un actor debería llevar a cabo una determinada actividad o desempeñar una determinada función ${ }^{77}$. De otra parte, un estándar de revisión consiste en un test que las cortes deben aplicar cuando revisan la conducta de un actor para determinar si efectivamente es responsable o no ${ }^{78}$.

De acuerdo con la definición de la Corte Suprema de Delaware, el deber de diligencia obliga a los administradores a actuar con el mismo "nivel de diligencia que normalmente una persona prudente $y$ diligente observaría en circunstancias similares"79. Es así como se quebranta el deber de diligencia siempre que los administradores actúen negligentemente ${ }^{80}$. En cambio, la BJR exime conductas negligentes de los mismos ${ }^{81}$, en el entendido de que los administradores responden en aquellos casos en que haya mediado culpa grave ${ }^{82}$. En últimas, la función de la BJR es crear un estándar de revisión menos estricto que el estándar de conducta creado a través del deber de diligencia ${ }^{83}$.

74 M. Aron Eisenberg, The Divergence of Standards of Conduct and Standards of Review in Corporate Law, 62 Fordham L. Rev., 437, 444-45 (1993).

75 T. Allen et ál., Function Over Form: A Reassessment of Standards of Review in Delaware Corporation Law, 56 Bus. Law, 1287, 1295-96 (2001).

76 Id.

77 Id.

78 Id.

79 Graham v. Allis-Chalmers Mfg. Co., 188 A.2d 125, 130 (Del. 1963).

80 Franklin A. Gevurtz, Corporation Law, 274 (2000).

81 Joy v. North, 692 F.2d 880, 885 (2d Cir. 1982).

82 F. A. Gevurtz, Corporation Law, 284-86 (2000).

83 M. Aron Eisenberg, Corporations and Other Business Organizations: Cases and Materials, 8th ed., 547 (2000); William T. Allen et ál., Realigning the Standard of Review of Director Due Care with Delaware Public Policy: A Critique of Van Gorkom and its Progeny as a Standard of Review Problem, 96 Nw. U. L. Rev., 449 (2002). 


\section{H. Justificación de la regla}

Si bien existen diversas justificaciones para la $\mathrm{BJR}^{84}$, creemos que estas pueden ser agrupadas en tres generales, esto es: alentar a los administradores para servir como tal ${ }^{85}$ y a tomar riesgos ${ }^{86}$; evitar la intervención judicial en las decisiones de negocios ${ }^{87}$; y preservar a la junta directiva como el cuerpo central de toma de decisiones en materia de gobierno corporativo ${ }^{88}$.

\section{Estimular a los administradores a ejercer su cargo y a tomar riesgos}

Respecto de estimular a los administradores tanto en su labor como a tomar riesgos, es preciso puntualizar que existe un interés general tanto de los accionistas como de la economía para que estos tomen riesgos de negocios ${ }^{89}$. Si los administradores supieran que sus decisiones van a estar sujetas a escrutinio judicial después de ser adoptadas, ellos estarían menos dispuestos a servir como tal, y quienes lo hicieran, serían excesivamente cuidadosos en su toma de decisiones ${ }^{90}$. Es del caso subrayar, sin embargo, que ser extremadamente cuidadoso en materia de negocios no es siempre una virtud, sino que puede llegar a ser, más bien, "un vicio costoso"91.

Las cortes reconocen que administradores competentes pueden tomar decisiones que en retrospectiva fueron imprudentes ${ }^{92}$. Si esas decisiones resultan en una pérdida significativa para la compañía,

84 Ver por ejemplo: Franklin A. Gevurtz, The Business Judgment Rule: Meaningless Verbiage or Misguided Notion? 67 S. Cal. L. Rev., 287, 304-21 (1994) (describiendo cuatro categorias); Kenneth B. Davis, Jr., Once More, The Business Judgment Rule, 2000 Wis. L. Rev., 573, 130 (2000) (describiendo cinco categorias).

85 Ver por ejemplo: Washington Bancorp v. Said, 812 F. Supp. 1256, 1267-68 (D.D.C. 1993).

86 Ver por ejemplo: Resolution Trust Corp. v. Blasdell, 930 F. Supp. 2d 417, 423 (D. Ariz. 1994); Air Line Pilots Ass'n, 717 F. Supp. at 582.

87 Ver por ejemplo: Dodge v. Ford Motor Co., 170 N.W. 668, 684 (Mich. 1919); In re J.P. Stevens \& Co. S'holders Litig., 542 A.2d 770, 780 (Del. Ch. 1988).

88 Ver por ejemplo: Michael P. Dooley \& E. Norman Veasey, The Role of the Board in Derivative Litigation: Delaware Law and the Current ALI Proposals Compared, 44 Bus. Law, 503, 522 (1989).

89 Paula J. Dalley, The Business Judgment Rule: What You Thought You Knew, 60 Consumer Fin L.Q. Rep., 24-25 (2006).

90 Id.

91 L. Herzel \& Leo Katz, Smith v. Van Gorkom: The Business of Judging Business Judgment, 41 Bus. Law. 1187, 1189 (1986).

92 Dennis J. Block et ál., The business judgment rule: fiduciary duties of corporate directors, 5th ed., 12 (1998). 
una regla legal que los haga fácilmente responsables por dicha pérdida disuadiría a las personas a actuar como administradores ${ }^{93}$. En consecuencia, la BJR, que dificulta declarar responsables a los administradores por decisiones imprudentes, estimula a personas competentes a convertirse en administradores, quienes de otra manera no lo harían por miedo a la responsabilidad personal en que podrían incurrir ${ }^{94}$.

Una vez los administradores están dispuestos a servir, deben tomar decisiones de negocios en favor de los accionistas ${ }^{95}$. Normalmente los accionistas quieren que los administradores tomen más riesgos de los que estos últimos quisieran asumir, dado que, en muchas ocasiones, los administradores no poseen ninguna o tienen muy pocas acciones de la compañía, de manera que reciben una muy baja o nula compensación por el riesgo asumido ${ }^{96}$. Una regla que imponga severamente responsabilidad sobre los administradores por decisiones imprudentes, aumentaría la tendencia a que estos se vuelvan adversos al riesgo $0^{97}$ y los disuadiría a adelantar negocios arriesgados, pero potencialmente lucrativos ${ }^{98}$.

De este modo, la BJR pretende alinear las actitudes frente al riesgo de los administradores con las de los accionistas, lo cual fomenta el objetivo de maximizar la riqueza de estos últimos ${ }^{99}$. En este punto es preciso puntualizar que el modelo anglosajón de gobierno corporativo está cimentado sobre la base de que el foco exclusivo de las prácticas de negocios ha de ser la maximización de la riqueza de los accionistas ${ }^{100}$.

93 Lyman P. Q. Johnson, Corporate Officers and The Business Judgment Rule, 60 Bus. Law., 439, 456 (2005).

94 Air Line Pilots Ass'n Int'l v. UAL Corp., 717 F. Supp. 575, 582 (N.D. Ill. 1989), aff'g, 897 F.2d 1394 (1990).

95 Lyman P. Q. Johnson, Corporate Officers and The Business Judgment Rule, 60 Bus. Law, 439, $456(2005)$.

96 Id.

97 Gagliardi v. Trifoods Int'l, Inc., 683 A.2d 1049, 1052 (Del. Ch. 1996).

98 Lyman P.Q. Johnson, Corporate Officers and The Business Judgment Rule, 60 Bus. Law, 439. 456 (2005).

99 Gagliardi v. Trifoods Int'l, Inc., 683 A.2d 1049, 1052-1053 (Del. Ch. 1996).

100 J. R. Macey \& Maureen O'Hara, The Corporate Governance of Banks, Fed. Res. Bank of N.Y. Econ. Pol'y R., 91 (2003). 


\section{J. Evitar el escrutinio judicial}

En relación con la no intervención judicial en las decisiones de los administradores, se debe subrayar que los jueces no son personas de negocios ${ }^{101} \mathrm{y}$, por tanto, no tienen la experiencia, el conocimiento o la información necesaria para tomar una decisión complicada de este tipo ${ }^{102} \mathrm{y}$, de igual forma, carecen del "sentido intangible" de comprender las circunstancias especificas con que se enfrenta una empresa ${ }^{103}$.

Además de lo anterior, los jueces sufren de "hindsight bias" (sesgo retrospectivo), que es una tendencia de estos a asignarle erróneamente una alta probabilidad a un evento de probable ocurrencia por el simple hecho de que terminó ocurriendo ${ }^{104}$. El respeto por la BJR impide que los jueces desconozcan el hecho de que la función del empresario es hacer frente a la incertidumbre y a los riesgos, de manera que una decisión que parecía ser razonada al momento de ser adoptada, puede parecer una "corazonada salvaje" años más tarde cuando se analiza en un contexto de conocimiento perfecto ${ }^{105}$.

A diferencia de los jueces ${ }^{106}$, los administradores que toman malas decisiones afrontan el riesgo de ser sancionados por los accionistas, quienes pueden votar para que estos dimitan, y de ser presionados por las fuerzas correctivas y competitivas del mercado ${ }^{107}$.

Dichas fuerzas de mercado incentivan a los administradores, principalmente a aquellos de compañías que cotizan en el mercado de valores, a actuar con debida diligencia en su toma de decisiones de negocios ${ }^{108}$. Si los administradores de este tipo de compañías actúan negligentemente, el precio de las acciones de la compañía caerá toda vez que los "inversionistas racionales solo le confiaran sus fondos a firmas en las que tengan confianza en su administración; firmas que son conocidas por tener administradores negligentes no atraerán

101 Dodge v. Ford Motor Co., 170 N.W. 668, 684 (Mich. 1919).

102 Daniel R. Fischel, The Corporate Governance Movement, 35 Vand. L. Rev., 1259, 1288 (1982).

103 D M. Branson, The Rule That Isn't a Rule-The Business Judgment Rule, 36 Val. U. L. Rev., 631637 (2002).

104 S M. Bainbridge, The Business Judgment Rule as Abstention Doctrine, 57 Vand. L. Rev., 83 114 (2004).

105 Joy v. North, 692 F.2d 880, 886 (2d Cir. 1982), cert. denied, 460 U.S. 1051 (1983).

106 Kamen v. Kemper Fin. Servs., Inc., 908 F.2d 1338, 1343 (7th Cir. 1990).

107 Kumpf v. Steinhaus, 779 F.2d 1323, 1325 (7th Cir. 1985).

108 Michael P. Dooley, Two Models of Corporate Governance, 47 Bus. Law, 461, 525 (1992). 
inversionistas o solo los atraerán a un precio descontado"109. En compañías cuyas acciones son altamente tranzadas, los inversionistas terminan descubriendo si sus administradores son negligentes o no, incluso en ausencia de pérdidas catastróficas, pues "inversionistas profesionales y asesores de inversión continuamente monitorean a las firmas y a sus administradores" ${ }^{\prime 10}$. Si el precio de las acciones de una compañía que cotiza en bolsa cae a un nivel lo suficientemente bajo, dicha compañía se puede convertir en blanco atractivo para una adquisición hostil, con lo cual los administradores pueden ser removidos de su cargo ${ }^{111}$. De este modo, las fuerzas del mercado inducen a los administradores a actuar diligentemente.

\section{K. Conservar a la junta directiva como último órgano de toma de decisión}

Por último, los administradores son los escogidos por los accionistas para tomar decisiones en nombre de la compañía ${ }^{112}$. Al limitar la revisión judicial, la BJR preserva el esquema de centralizar la autoridad en la junta directiva ${ }^{113}$. En cambio, si se les permite a los accionistas impugnar fácilmente las decisiones adoptadas por los administradores, la junta directiva le transferiría el estatus de última autoridad en la toma de decisiones a cualquier accionista que esté dispuesto a interponer una demanda ${ }^{114}$. De esta forma, la BJR preserva la autoridad de los administradores en la toma de decisiones sobre los accionistas y la intervención judicial ${ }^{115}$.

\section{Críticas a la regla}

No obstante los beneficios de la BJR, esta ha sido igualmente criticada. Existen críticas que parten de argumentos de costo - beneficio,

109 C. Samuel Bjerre, Evaluating the New Director Exculpation Statutes, 73 Cornell L. Rev., 786, 798 (1988)

110 Id. at 799.

111 Id. at 798-800

112 Paula J. Dalley, The Business Judgment Rule: What You Thought You Knew, 60 Consumer Fin L.Q. Rep., 24. 25 (2006)

113 Michael Dooley \& E. Norman Veasey, The Role of the Board in Derivative Litigation: Delaware Law and the Current ALI Proposals Compared, 44 Bus. Law, 503, 522 (1989).

114 M. Dooley, Not in the Corporation's Best Interests, A.B.A.J., May 1992, at 45.

115 In reFuqua Indus., Inc. S'holderLitig., No. Civ. A. 11974, 1997 WL 257460, at *12 (Del. Ch. May 13, 1997). 
los cuales, sirven como contraargumentos a los análisis reseñados en la sección anterior. En términos generales, algunos críticos señalan que incrementar la responsabilidad de los administradores puede generar muchos más beneficios de lo que anteriormente se pensaba, al punto de que el resultado del análisis de costo - beneficio puede favorecer el incremento de responsabilidad de los administradores.

Después de los escándalos de Enron y de Worldcom, en los que se ha dicho que la actuación negligente de los administradores fue una causa significante en el colapso de dichas compañías ${ }^{116}$, algunos doctrinantes han señalado que la negligencia de los administradores puede causar daños muy serios, de manera que al incrementar la responsabilidad de los mismos se podría llegar a disuadir su conducta negligente ${ }^{117}$. Debido a que un colapso financiero puede llegar a causar un daño de enormes magnitudes a la sociedad, se debería aumentar la responsabilidad de los administradores en aras de evitar, en un futuro, colapsos de ese tipo ${ }^{118}$. Si bien el incremento de responsabilidad de los administradores puede acarrear algunos costos, hay quienes afirman que "dada la importancia de la responsabilidad legal para la regulación de la conducta de los administradores, dichos costos tienen que ser absorbidos" al poderse prevenir futuras crisis financieras ${ }^{119}$.

De forma similar, otros comentaristas apuntan a que los escándalos de Enron y de WorldCom fueron causados en gran medida porque los administradores no actuaron de buena $\mathrm{fe}^{120}$. Para solventar dicho problema, se arguye que Delaware debería establecer una concepción más robusta del deber de actuar de buena fe, con lo cual se cambiaría el comportamiento de los administradores y se incentivaría a estos a cumplir con sus deberes fiduciarios ${ }^{121}$. De acuerdo con esta posición, presumiblemente, los mencionados beneficios serían mayores a los costos asociados con incrementar la responsabilidad de los administradores ${ }^{122}$.

\footnotetext{
116 L. M. Fairfax, Spare the Rod, Spoil the Director? Revitalizing Directors' Fiduciary Duty Through Legal Liability, 42 Hous. L. Rev., 393, 400 (2005).

117 Id. at $394-396$.

118 Id. at 449.

119 Id. at 455.

120 Hilary A. Sale, Delaware’s Good Faith, 89 Cornell L. Rev., 456, 461-462 (2004).

121 Id. at 488-494.

122 T. M. Aman, Cost-Benefit Analysis of the Business Judgment Rule: A Critique in Light of the Financial Meltdown, 74 Alb. L. Rev., 1. 21 (2011).
} 
De otra parte, doctrinantes han criticado la BJR señalando la diferencia en el tratamiento que le otorga el ordenamiento jurídico a los administradores frente a otros profesionales como pueden ser médicos y abogados ${ }^{123}$. Aunque no existe ninguna justificación de política pública para sustentar dicha diferenciación, las actuaciones de los administradores son evaluadas por el estándar de la culpa grave, mientras que los demás profesionales son medidos a través de la simple culpa ${ }^{124}$. Quienes apoyan dicha posición, señalan que debería existir un estándar universal aplicable tanto a los administradores como a los demás profesionales, el cual debería ser determinado después de realizar un análisis de costo - beneficio ${ }^{125}$.

Así las cosas, los argumentos en favor de la BJR enfatizan los costos de incrementar la responsabilidad de los administradores, mientras que las críticas que se mencionaron anteriormente responden a dichos argumentos haciendo hincapié en los beneficios. Si bien dichas críticas sirven como contraargumentos a las razones que fundamentan la BJR, no es menos cierto que aquellas no las desaprueban del todo ${ }^{126}$. Con el fin de contrarrestar las alegaciones en favor de la BJR, sería necesario demostrar que los beneficios de aumentar la responsabilidad de los administradores sobrepasan los costos, para lo cual sería necesario catalogar todos los costos y beneficios asociados, darles un valor y hacer los cálculos respec$\operatorname{tivos}^{127}$. De otra manera, dichas críticas no podrían llevar, por sí mismas, a una conclusión sustentando una u otra posición ${ }^{128}$. Dada la complejidad, e incluso la imposibilidad, de realizar objetivamente cálculos de tales magnitudes ${ }^{129}$, resulta muy complicado demostrar las críticas a la BJR a partir de un análisis de costo - beneficio.

123 F. A. Gevurtz, The Business Judgment Rule: Meaningless Verbiage or Misguided Notion?, 67 S. Cal. L. Rev., 287, 295 (1993).

124 Id. at 304-05.

125 Id. at 318 .

126 Todd M. Aman, Cost-Benefit Analysis of the Business Judgment Rule: A Critique in Light of the Financial Meltdown, 74 Alb. L. Rev., 1. 23 (2011).

127 D.C. Langevoort, The Human Nature of Corporate Boards: Law, Norms, and the Unintended Consequences of Independence and Accountability, 89 Geo. L.J., 797, 829 (2001).

128 Id.

129 D. Kennedy, Law and Economics from the Perspective of Critical Legal Studies, in Peter Newman, Ed., 2 The New Palgrave Dictionary of Economics and the Law, 465, 469-70 (1998). 


\section{Aplicación de la BJR a administradores de bancos}

Los bancos tienen características particulares que los hacen distintos a otros tipos de negocios: a diferencia de otras compañías, los bancos tienen muy poco patrimonio; mientras que otras compañías se financian a través de acciones y de emisión de deuda, los bancos generalmente financian el $90 \%$ de sus operaciones por medio de deuda; los pasivos de los bancos generalmente son depósitos mientras que los activos son préstamos con términos de duración más prolongados; los bancos normalmente tienen a la mano un pequeño porcentaje de los fondos depositados, puesto que la mayoría de estos son reutilizados para hacer otros préstamos. De este modo, si un gran porcentaje de sus clientes quisiera retirar sus fondos simultáneamente, incluso el banco más solvente quebraría ${ }^{130}$.

Las posibles consecuencias adversas que pueden generar en la economía y en la sociedad la estructura de capital de los bancos, justifican una especial regulación y tratamiento para con estos. Una de las formas más sencillas para incrementar la regulación de los bancos es reduciendo la esfera de autonomía de sus administradores, comparación con la que gozan los administradores de otros negocios. Es decir, haciendo más débil la aplicación de la BJR en temas bancarios ${ }^{131}$.

Dicha tendencia se ha visto reflejada en decisiones de distintas cortes en los Estados Unidos. En Hun v. Cary, los administradores del New York Central Savings Bank fueron demandados por haber aprobado la construcción de nuevas oficinas, pese a que el banco en ese momento era prácticamente insolvente ${ }^{132}$. Aunque los administradores del banco alegaron que dicha construcción podría atraer nuevos negocios e incrementar la rentabilidad del banco, la Corte los declaró responsables porque la construcción de una nueva sede para el banco no tenía potencial de ser un negocio rentable, sino que, por el contrario, pudo haberlo ahogado en deudas ${ }^{133}$.

En el mismo sentido, en Litwin v. Allen los directores de Guaranty Trust aprobaron la compra de bonos de una compañía llamada

130 J. R. Macey \& Maureen O’Hara, The Corporate Governance of Banks, Fed. Res. Bank of N.Y. Econ. Pol'y R., 91, 97 (2003).

$131 \mathrm{Id}$.

132 Hun v. Cary, 82 N.Y., 65, 66-77 (1880).

133 Id. at $70-79$. 
Missouri Pacific a una tasa de interés fija ${ }^{134}$. La compañía matriz de Missouri Pacific, Alleghany Corporation, acordó una opción de compra de los bonos durante los primeros seis meses del negocio. No obstante lo anterior, Guaranty Trust no acordó, recíprocamente, una opción de venta en el supuesto de que el precio de los bonos cayera durante esos seis meses. Finalmente, el precio de los bonos se redujo y Alleghany Corporation no ejerció la opción de compra. La Corte declaró responsables a los directores al considerar que habían sido negligentes por no haber acordado una opción de venta y por haber puesto a Guaranty Trust en una situación en la que debía soportar todo el riesgo de pérdida sin tener ninguna posibilidad de ganancia ${ }^{135}$.

Tanto de uno como del otro caso, se puede inferir que en el evento de que una decisión adoptada por los administradores de bancos no sea potencialmente rentable, estos podrán estar sujetos a responsabilidad ${ }^{136}$. La definición de qué es rentable y qué no lo es, resulta muy complicada toda vez que, por ejemplo, en Litwin v. Allen los bonos comprados por los directores pagaban una tasa de interés fija, lo cual, en principio, debería ser considerado como rentabilidad ${ }^{137}$.

En otros casos, en vez de condenar a los administradores de bancos por tomar decisiones que no tenían posibilidad de ganancia, lo han hecho por decisiones que son "muy arriesgadas", incluso cuando existía un alto potencial de ganancia ${ }^{138}$. Tal es el caso de FDIC v. Robertson, en el que la Corte encontró responsables a los administradores por extender un crédito a un nuevo negocio que no poseía un historial de rentabilidad, ni estados financieros adecuados y no existía un programa de reembolso del crédito ${ }^{139}$. Sin embargo, en FDIC v. Stanley la Corte no declaró responsables a los administradores que aprobaron un crédito a una nueva empresa sin historial de rentabilidad. La Corte señaló que mientras exista una garantía para el crédito y la situación financiera de quien solicita

134 Litwin v. Allen, 25 N.Y.S. 2d 667, 691-93 (1940).

135 Id. at $690-98$.

136 P. A. McCoy, The Notional Business Judgment Rule in Banking, 44 Catholic U. L. Rev., 1031, 1037-40 (1995).

137 Id. at 1039.

138 J. Canfield. The Evolution of a More Stringent Business Judgment Rule in Banking: The Minimilization of Director Deference. 6 U.C. Davis Bus. L.J., 17 (2006).

139 FDIC v. Robertson, No. 87-2623-S, 1989 U.S. Dist. Lexis 9292 at 16-18 (D. Kan. July 24, 1989) 
el crédito esté mejorando, los administradores no serán declarados responsables ${ }^{140}$. De este modo, no todas las cortes han condenado a los administradores de bancos por prestar dinero a nuevas empresas que no tienen historial de ser rentables ${ }^{141}$.

Asimismo, también existen decisiones contradictorias respecto de la responsabilidad de los administradores de bancos por otorgar créditos sin suficiente garantía. Por ejemplo, en FDIC v. Wheat, la Corte declaró responsable al administrador de un banco por, entre otros, haber actuado negligentemente al haber aprobado un crédito en el que existía únicamente una garantía personal ${ }^{142}$. En contraposición, en Starrels v. First Nat'l Bank of Chicago la Corte decidió que a pesar de que el crédito aprobado por el banco carecía de garantía, la decisión del administrador de extender el crédito recaía dentro de la $\mathrm{BJR}^{143}$. En el mismo sentido, en Noble v. Baum la Corte concluyó que la decisión de otorgar un crédito sin que el prestatario del mismo tuviera patrimonio, estaba protegida por la $\mathrm{BJR}^{144}$.

Pese a que no existe uniformidad respecto de la aplicación de la BJR en temas bancarios, lo que sí está claro es que las cortes en los Estados Unidos tienden a examinar con mayor rigor y cuidado las decisiones de los administradores de bancos en comparación con las que son adoptadas por administradores de otro tipo de compañías $^{145}$.

140 FDIC v. Stanley, 770 F. Supp. 1281, 1293-1304 (N.D. Ind. 1991).

141 J. Canfield. The Evolution of a More Stringent Business Judgment Rule in Banking: The Minimilization of Director Deference. 6 U.C. Davis Bus. L.J., 17 (2006).

142 FDIC v. Wheat, 970 F.2d at 129.

143 Starrels v. First Nat'l Bank of Chicago, 870 F.2d 1168 1171-72 (7th Cir. 1989).

144 Nobel v. Baum, No. CV 890265920 S, 1991 Conn. Super. Lexis 1231, at 7, 40-41 (Conn. Super. Ct. May 6, 1991).

145 J. Canfield. The Evolution of a More Stringent Business Judgment Rule in Banking: The Minimilization of Director Deference. 6 U.C. Davis Bus. L.J., 17 (2006). 


\section{CONCLUSIóN}

La BJR es una de regla del common law utilizada con el propósito de reducir las acciones de responsabilidad iniciadas por los accionistas contra los administradore ${ }^{146}$, respetando las diversas decisiones adoptadas por estos, bien sean convenientes o no para aquellos.

No obstante haber sido formulada como una regla, la BJR es un concepto legal de constante evolución ${ }^{147}$ que se ha venido adaptando a los tiempos y a las distintas circunstancias. Lo anterior se puede inferir, por ejemplo, a partir de los requisitos adicionales que incorporaron las cortes de Delaware durante los años ochenta a la aplicación de la BJR en operaciones de M\&A y que surgieron como respuesta a la proliferación de adquisiciones hostiles en esa década en los Estados Unidos.

Si bien muchos países del derecho continental europeo no han adoptado aún la BJR como una presunción dentro de su legislación, no es menos cierto que un gran número de criterios utilizados por las cortes de los Estados Unidos en relación con la BJR resultan ser aplicables en dichos países y, sin duda, sirven para orientar a jueces, árbitros y abogados en casos relacionados con responsabilidad de los administradores.

146 D. M. Two Models of Corporate Governance. 47 Bus. Law., 461, 469-70 (1992).

147 Bayless Manning, Reflections and Practical Tips on Life in the Boardroom After Van Gorkom, 41 Bus. Law, 1, 1 (1985). 


\section{BIBLIOGRAFÍA}

\section{Doctrina}

American Law Institute, Principles of Corporate Governance: Analysts and Recommendations (1994) (Principles).

B. Manning, Reflections and Practical Tips on Life in the Boardroom After Van Gorkom, 41 Bus. Law, 1 (1985).

C. Guerrero Trevijano, La Business Judgment Rule en los Procesos de $M \& A$. Documentos de Trabajo del Departamento de Derecho Mercantil, Universidad Complutense (2010).

C. Samuel Bjerre, Evaluating the New Director Exculpation Statutes, 73 Cornell L. Rev., 786 (1988).

D. A. Drexler, Lewis S. Black, \& A. Gilchrist Sparks, Delaware Corporation Law and Practice (2010).

D. C. Langevoort, The Human Nature of Corporate Boards: Law, Norms, and the Unintended Consequences of Independence and Accountability, 89 Geo. L. J., 797 (2001).

D. Fischel, The Corporate Governance Movement, 35 Vand. L. Rev., 1259 (1982).

D. J. Block et ál., The Business Judgment Rule-Fiduciary Duties of Corporate Directors, 5th ed., 6 (1998).

D. Kennedy, Law and Economics from the Perspective of Critical Legal Studies, in: Peter Newman, Ed., 2 The New Palgrave Dictionary of Economics and the Law, 465 (1998).

D. M. Branson, The Rule That Isn't a Rule - The Business Judgment Rule, 36 Val. U. L. Rev., 631 (2002).

D. M., Two Models of Corporate Governance, 47 Bus. Law., 461 (1992).

E. S. Miller \& Thomas E. Rutledge, The Duty of Finest Loyalty and Reasonable Decisions: The Business Judgment Rule in Unincorporated Business Organizations? 30 Del. J. Corp. L. 343 (2005).

F. A. Gevurtz, Corporation Law, St. Paul, Minn. West, (2000).

, The Business Judgment Rule: Meaningless Verbiage or Misguided Notion?, 67 S. Cal. L. Rev., 287 (1994).

G. Subramanian, Fixing Freezouts, 115 Yale Law Journal (2005).

H. A. Sale, Delaware’s Good Faith, 89 Cornell L. Rev., 456 (2004).

J. Canfield. The Evolution of a More Stringent Business Judgment Rule in Banking: The Minimilization of Director Deference, 6 U.C. Davis Bus. L. J., 17 (2006).

J. R. Macey \& Maureen O'Hara, The Corporate Governance of Banks, Fed. Res. Bank of N.Y. Econ. Pol'y R., 91 (2003).

K. B. Davis, Jr., Once More, The Business Judgment Rule, 2000 Wis. L. Rev., 573 (2000). 
L. E. Strine, Jr., Delaware's Corporate-Law System: Is Corporate America Buying an Exquisite Jewel or a Diamond in the Rough? A Response to Kahan \& Kamar's Price Discrimination in the Market for Corporate Law, 86 Cornell L. Rev., 1257 (2001).

L. Fairfax, Spare the Rod, Spoil the Director? Revitalizing Directors' Fiduciary Duty Through Legal Liability, 42 Hous. L. Rev., 393 (2005).

L. Herzel \& Leo Katz, Smith v. Van Gorkom: The Business of Judging Business Judgment, 41 Bus. Law., 1187 (1986).

L. Johnson, Corporate Officers and The Business Judgment Rule 60 Bus. Law. 439 (2005). , The Modest Business Judgment Rule. 55 Bus. Law. 625 (2000).

M. Aron Eisenberg, Corporations and Other Business Organizations: Cases and Materials, 8th ed., New York, West, 547 (2000).

M. Aron Eisenberg, The Divergence of Standards of Conduct and Standards of Review in Corporate Law, 62 Fordham L. Rev., 437 (1993).

M. Dooley, Not in the Corporation's Best Interests, A.B.A. J. (May 1992).

M. Dooley, Two Models of Corporate Governance, 47 Bus. Law, 461 (1992).

M. Dooley \& E. Norman Veasey, The Role of the Board in Derivative Litigation: Delaware Law and the Current ALI Proposals Compared, 44 Bus. Law, 503 (1989).

M. McMurray, Historical Perspective on the Duty of Care, the Duty of Loyalty, and the Business Judgment Rule, 40 Vand. L. Rev., 607 (1987).

P.A. McCoy, The Notional Business Judgment Rule in Banking, 44 Catholic U. L. Rev., 1031 (1995).

P.J. Dalley, The Business Judgment Rule: What You Thought You Knew, 60 Consumer Fin L.Q. Rep., 24 (2006).

Principles of Corporate Governance: Analysis and Recommendations (1994).

P. V. Letsou, Implications of Shareholder Diversification on Corporate Law and Organization: The Case of the Business Judgment Rule, 77 Chi.-Kent L. Rev., 179, (2001).

R. Franklin Balotti \& Joseph Hinsey IV, Director Care, Conduct, and Liability: The Model Business Corporation Act Solution, 56 Bus. Law, 35-56 (2000).

S. M. Bainbridge, The Business Judgment Rule as Abstention Doctrine, 57 Vand. L. Rev., 83 (2004).

Smith v. Van Gorkom: The Business of Judging Business Judgment, 41 Bus. Law, 1187 (1986).

S. Worthing, Reforming Director's Duties, 64 Modern Law Review, No. 3 (2001).

T. Allen et ál., Function Over Form: A Reassessment of Standards of Review in Delaware Corporation Law, 56 Bus. Law, 1287 (2001).

T. M. Aman, Cost-Benefit Analysis of the Business Judgment Rule: A Critique in Light of the Financial Meltdown, 74 Alb. L. Rev., 1. 21 (2011).

W.T. Allen et ál., Realigning the Standard of Review of Director Due Care with Delaware Public Policy: A Critique of Van Gorkom and its Progeny as a Standard of Review Problem, 96 Nw. U. L. Rev., 449 (2002). 


\section{Casos}

Air Line Pilots Ass'n Int'l v. UAL Corp., 717 F. Supp. 575 (N.D. Ill. 1989).

Aronson v. Lewis, 473 A.2d 805 (Del. 1984).

Brehm v. Eisner, 746 A.2d 244 (Del. 2000).

Cede v. Technicolor Inc., 634 A.2d 361 (Del. 1993).

Cede \& Co. v. Technicolor, Inc., 634 A.2d 345 (Del. 1993).

Crescent/Mach I Partners, L.P. v. Turner, 846 A.2d 963 (Del. Ch. 2000).

Dodge v. Ford Motor Co., 170 N.W. 668 (Mich. 1919).

Doskocil Cos. v. Griggy, CA, 10095, 10106-08, 10116, 1988 Del. Ch. Lexis 113 (Aug. 18, 1988).

Edgar v. MITE Corp., 457 U.S. 624 (1982).

Emerald Partners v. Berlin, 726 A.2d 1215 (Del. 1999).

FDIC v. Robertson, No. 87-2623-S, 1989 U.S. Dist. Lexis 9292 (D. Kan. July 24, 1989).

FDIC v. Stanley, 770 F. Supp. 1281 (N.D. Ind. 1991).

FDIC v. Wheat, 970 F.2d.

Gagliardi v. Trifoods Int'l, Inc., 683 A.2d 1049 (Del. Ch. 1996).

Graham v. Allis-Chalmers Mfg. Co., 188 A.2d 125 (Del. 1963).

Henley Group, Inc. v. Santa Fe Southern Pacific Corp., CA 9569, 1988 Del. Ch. Lexis 32 (Mar. 11, 1988).

Hun v. Cary, 82 N.Y. 65 (1880).

In reFuqua Indus., Inc. S'holderLitig., No. CIV. A. 11974, 1997 WL 257460 (Del. Ch. May 13, 1997).

In re J.P. Stevens \& Co. S'holdersLitig., 542 A.2d 770 (Del. Ch. 1988).

In re Time, Inc. S'holdersLitig., 571 A.2d 1140 (Del. 1989).

In re Walt Disney Co. Derivative Litig., 825 A.2d 275 (Del. Ch. 2003).

Ivanhoe Partners v. Newmont Mining Corp., 535 A.2d 1334 (Del. 1987).

Joy v. North, 692 F.2d 880 (2d Cir. 1982).

Kahn v. Lynch Commun. Sys., Inc., 638 A.2d 1210 (Del. 1994).

Kamen v. Kemper Fin. Servs., 908 F.2d 1338 (7th Cir. 1990).

Kaplan v. Centex Corp., 284 A.2d 119 (Del. Ch. 1971).

Katz v. Chevron Corp., 27 Cal. Rptr.2d 681 (Cal. Ct. App. 1994).

Kumpf v. Steinhaus, 779 F.2d 1323 (7th Cir. 1985).

Litwin v. Allen, 25 N.Y.S.2d 667 (1940).

McMullin v. Beran, 765 A.2d 910 (Del. 2000). 
Miller v. American Telephone \& Telegraph, 507 F.2d 759 (3rd Cir. 1974).

Nobel v. Baum, No. CV 890265920 S, 1991 Conn. Super. Lexis 1231 (Conn. Super. Ct. May 6, 1991).

Paramount Communications Inc. v. QvC Network Inc., 637 A. 2d 34 (Del. 1994).

Paramount Commc'ns Inc. v. Time Inc., 1989 Del.Ch. Lexis 77 (July 14, 1989)

Parnes v. Bally Entertainment Corp, 722 A.2d 1243 (Del. 1999).

Penn Mart Realty Co. v. Becker, 298 A.2d 349 (Del.Ch.1972).

Radol v. Thomas, 772 F.2d 244 (6th Cir. 1985).

Resolution Trust Corp. v. Blasdell, 930 F. Supp. 2d 417 (D. Ariz. 1994).

Revlon, Inc. v. MacAndrews \& Forbes Holdings, Inc., 506 A.2d 182 (Del.1986).

Smith v. Van Gorkom, 488 A.2d 858 (Del. 1985).

Starrels v. First Nat'l Bank of Chicago, 870 F.2d 1168 (7th Cir. 1989).

Unocal Corp. v. Mesa Petroleum Co., 493 A.2D 946 (Del.1985).

Van de Walle v. Unimation, Inc., [1990-1991 Transfer Binder] Fed. Sec. L. Rep. (сCH) (Del. Ch. Mar. 6, 1991).

Washington Bancorp v. Said, 812 F. Supp. 1256 (D.D.C. 1993).

Weinberger v. UOP, Inc., 457 A.2d 701 (Del. 1983). 
